\title{
Expression and localization of special AT-rich sequence binding protein 2 in murine molar development and the pulp-dentin complex of human healthy teeth and teeth with pulpitis
}

\author{
LINA HE* , HUIMEI LIU* , LEI SHI, SHUANG PAN, XU YANG, LIN ZHANG and YUMEI NIU \\ Department of Endodontics, The First Affiliated Hospital of \\ Harbin Medical University, Harbin, Heilongjiang 150001, P.R. China
}

Received September 18, 2016; Accepted June 13, 2017

DOI: $10.3892 /$ etm.2017.4980

\begin{abstract}
Special AT-rich sequence binding protein2(SATB2) is a member of the special family of AT-rich binding transcription factors and has a critical role in osteoblast differentiation and craniofacial patterning. However, the expression and distribution of SATB2 in tooth development is largely unknown. The aim of the present study was to detect the expression and distribution of SATB2 during murine molar development and, in human healthy teeth and teeth with pulpitis using immunohistochemistry. Molars were obtained from Kunming mice at embryonic day (E) 13.5, E14.5, E16.5 and E18.5, and postnatal day (P) 1, P5 and P7. In addition, 20 human teeth (10 healthy and 10 teeth with pulpitis) were obtained from young adult patients (age, $24.90 \pm 1.65$ years) who were scheduled for routine extraction. Immunohistochemical analyses were performed to detect the expression and distribution of SATB2. The present results revealed that SATB2 exhibits a spatiotemporal expression pattern in murine molar development and was expressed in odontoblasts, predentin, dental pulp cells and the blood vessels in human teeth. These findings suggested that SATB2 may have an important role in odontoblast differentiation and dentin matrix mineralization during tooth development.
\end{abstract}

\section{Introduction}

Tooth development is a complex process that involves regulated and reciprocal interactions between the dental epithelium and the neural crest-derived mesenchyme (1).

Correspondence to: Dr Yumei Niu, Department of Endodontics, The First Affiliated Hospital of Harbin Medical University, 143 Yiman Street, Harbin, Heilongjiang 150001, P.R. China E-mail: yumeiniu@163.com

\section{${ }^{*}$ Contributed equally}

Key words: special AT-rich sequence binding protein 2, tooth development, odontoblast differentiation, dentin formation
The tooth germ forms at the bud stage, undergoes folding morphogenesis and develops into the cap stage. During the bell stage, the tooth crown forms and in the late bell stage, the epithelial and mesenchymal cells differentiate into ameloblasts and odontoblasts to form the enamel and dentin (2). During tooth development, various biological molecules are involved in regulating cell differentiation and tooth morphogenesis (3-6), including bone morphogenetic proteins (7) and transforming growth factor (8), which have important roles in osteoblast differentiation and bone formation (9). However, the precise mechanism of tooth development remains to be fully elucidated and other molecules may be associated with this process.

Special AT-rich sequence binding protein 2 (SATB2) is a member of the special family of AT-rich binding transcription factors that has a pivotal role in craniofacial patterning and has been identified as a marker of osteogenic differentiation $(10,11)$. SATB2 binds to nuclear matrix-attachment regions (MARs) (12). Studies have shown that SATB2 may regulate bone sialoprotein (BSP) and osteocalcin (OCN) expression by enhancing the activity of runt-related transcription factor 2 (Runx2) and activating transcription factor 4, which activates Runx2-dependent osteoblast differentiation $(11,13,14)$.

SATB2 is able to repress the expression of an inhibitor of bone formation and regulator of branchial arch patterning, homeobox a2 (Hoxa2), in osteoblasts, in a MAR-dependent manner (15). These findings suggest that SATB2 is associated with the regulation of BSP and OCN expression, and the repression of Hoxa2 expression to initiate osteoblast differentiation. Therefore, SATB2 may act as a transcriptional node that regulates the activity of transcriptional complexes and indirectly modulates the expression of genes that control osteoblast differentiation and maturation (15). Furthermore, SATB2 has been associated with craniofacial development and antagonizing bone formation (10). Therefore, it was hypothesized that SATB2 may have a potential role and interact in tooth development. The purpose of the present study was to investigate the expression and distribution of SATB2 during murine molar development, and in human healthy teeth and teeth with pulpitis using immunohistochemistry. 


\section{Materials and methods}

Preparation of tissue sections. All experiments were approved by the Ethics Committee of the First Affiliated Hospital of Harbin Medical University (Harbin, China) and informed written consent was provided from all patients involved in the present study. Embryos and postnatal mice were obtained by mating male and female Kunming mice (Nanjing Institute of Medicine and Biology, Nanjing, China). A total of 45 (male to female ratio, 1:2) 10-week-old Kunming mice (weight, 20-25 g) were housed at $4^{\circ} \mathrm{C}$ with $50 \%$ humidity in a 12 -h light/dark cycle, and fed a standard rodent diet with free access to water. Embryonic day 0.5 (E0.5) was assigned at $12 \mathrm{pm}$ on the day of the appearance of the vaginal plug, whereas $12 \mathrm{pm}$ on the day of birth was regarded as postnatal day 0.5 (P0.5). A total of 3 pregnant mice from each time point (E13.5, E14.5, E16.5 and E18.5) and 3 postnatal mice from different mothers and periods (P1, P5 and P7) were sacrificed and the embryos were removed from the pregnant mice (16). The heads (E13.5 and E14.5) and mandibles (E16.5, E18.5, P1, P5 and P7) of mice were dissected, and fixed in buffered $4 \%$ paraformaldehyde for $24 \mathrm{~h}$ at $4^{\circ} \mathrm{C}$. The mandibles (E18.5, P1, P5 and P7) were then decalcified in $10 \%$ EDTA/PBS solution for $24 \mathrm{~h}$ at room temperature.

A total of 20 fully developed and erupted third molars with closed apices were obtained from young adult patients (12 males and 8 females; age, $24.90 \pm 1.65$ years), enrolled between July and September 2015, for whom routine extraction was scheduled. The teeth included 10 healthy teeth and 10 teeth with pulpitis from patients who had obvious symptoms of pulpitis. The teeth were immediately placed into $0.1 \mathrm{M}$ PBS following extraction and washed three times. One third of the apical root was removed using diamond burs. Tooth samples were fixed with $4 \%$ paraformaldehyde in $0.1 \mathrm{M}$ PBS for $48 \mathrm{~h}$ at $4^{\circ} \mathrm{C}$, followed by demineralization for 6-9 weeks in $10 \%$ EDTA at room temperature.

All tissue samples obtained from mouse heads, mandibles and teeth were dehydrated through a graded alcohol series $(100$, $100,95,95,80$ and $70 \%$ ), embedded in paraffin and sectioned (5 $\mu \mathrm{m}$ thickness) for histology and immunohistochemistry.

Histology. The sample sections were deparaffinized in xylene, rehydrated through a gradient of ethanol solutions $(100,100,95,95,80$ and $70 \%)$, stained with hematoxylin and eosin at room temperature and observed under a light microscope at magnification, $\mathrm{x} 200$ or $\mathrm{x} 40$.

Immunohistochemistry. Immunohistochemical analyses were performed using the Instant Streptavidin-biotin Complex (SABC) kit (cat. no. SA1030; Bioss, Beijing, China) according to the manufacturer's protocol. To suppress endogenous peroxidase activity, deparaffinized sections were treated with $100 \mu 1$ $3 \% \mathrm{H}_{2} \mathrm{O}_{2}$ at room temperature for $10 \mathrm{~min}$. Sections were blocked in $5 \%$ normal goat serum for $1 \mathrm{~h}$ at room temperature and incubated with SATB2 primary antibodies (rabbit anti-mouse, cat. no. ab69995, diluted 1:100; Abcam, Cambridge, MA USA) overnight at $4{ }^{\circ} \mathrm{C}$. Incubation in PBS instead of primary antibodies served as the negative control. Sections were rinsed in PBS with Tween-20 (PBST) and incubated with biotinylated secondary antibodies (anti-rabbit IgG, cat. no. sc-2040, diluted
1:400; Santa Cruz Biotechnology, Inc., Dallas, TX, USA) for $45 \mathrm{~min}$ at room temperature. Subsequently, sections were washed three times in PBST, incubated in SABC for $30 \mathrm{~min}$ at room temperature and stained with $100 \mu 1$ 3,3'-diaminobenzidine solution (diluted 1:25) at room temperature for $\sim 1 \mathrm{~min}$ until brown coloration was detected. Slides were rinsed with distilled water and then counterstained at room temperature with hematoxylin for $1 \mathrm{~min}$ and observed under a light microscope at magnification, $\mathrm{x} 200$ or $\mathrm{x} 40$.

\section{Results}

Hematoxylin and eosin staining and immunostaining was used to determine the morphogenesis and structure of the first murine molars from E13.5-P7. The tooth germ underwent various development stages including the bud, cap and bell stages.

Expression of SATB2 in developing stages of murine molar development. At the bud stage (E13.5), the dental lamina was thickened and invaginated into the underlying mesenchyme. SATB2 was expressed in tooth bud cells and the underlying mesenchyme (Fig. 1A). During the cap stage (E14.5), the enamel organ appeared to have undergone further proliferation and differentiation. SATB2 expression was also detected in the enamel organ and mesenchyme (Fig. 1B). At the early bell stage (E16.5), SATB2-positive staining was exhibited in the inner and outer enamel epithelium, and the stratum intermedium; however, no SATB2 expression was indicated in the stellate reticulum. Positive staining for SATB2 expression was also indicated in the enamel knot (Fig. 1C). At the late bell stage (E18.5), positive immunostaining was observed in odontoblasts and dental papilla cells. The differentiating ameloblasts, stratum intermedium and inner enamel epithelium were all stained positively (Fig. 1D).

The expression of SATB2 was determined in postnatal murine tooth germs at P1 and P5 (Fig. 2). At P1 and P5, SATB2-positive staining was exhibited in the ameloblasts, and intense positive staining was observed in the odontoblasts and dental pulp cells (Fig. 2A and C). Particularly in the odontoblasts, SATB2 expression was identified in nuclei, which were potently stained, whereas in the ameloblasts, SATB2 expression was indicated predominantly in the cytosol and partially in nuclei (Fig. 2B and D). At P7, when mineralized tissue was gradually deposited, SATB2 expression was still detected in the ameloblasts and dental pulp cells (Fig. 3A). Furthermore, basic completion of the crown morphogenesis was observed and early root development was indicated. On the pulpal side of root dentin, odontoblasts were lined in a row. SATB2 expression was still potently expressed in the odontoblasts in the crown and in the root (Fig. 3B and C). Notably, SATB2-positive staining of odontoblasts in the root was more potent compared with that in the crown.

SATB2 expression in the pulp-dentin complex of human healthy teeth and teeth with pulpitis. Hematoxylin-eosin staining revealed normal histological structures for the samples of 10 healthy teeth. A total of 10 teeth with pulpitis were histologically confirmed. Immunohistochemical results showed that SATB2 was primarily localized in the odontoblasts and 


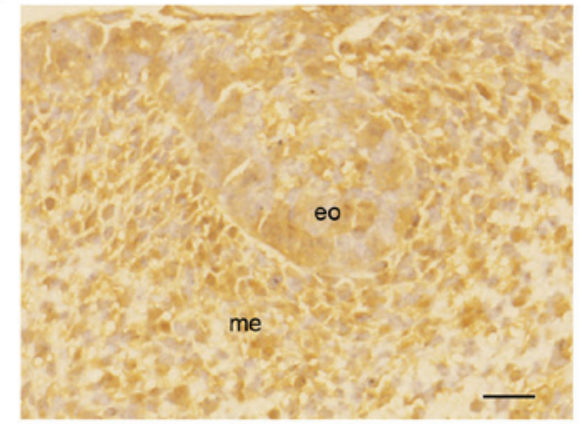

C

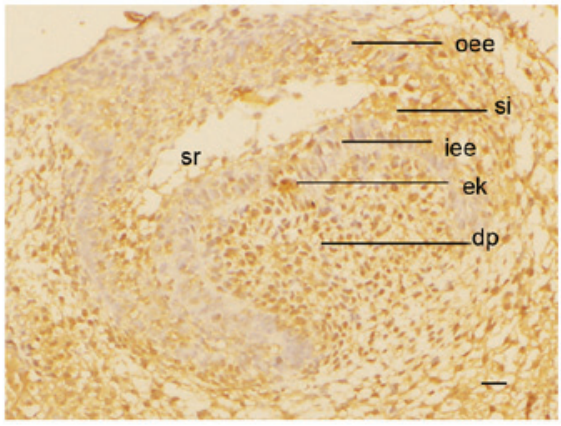

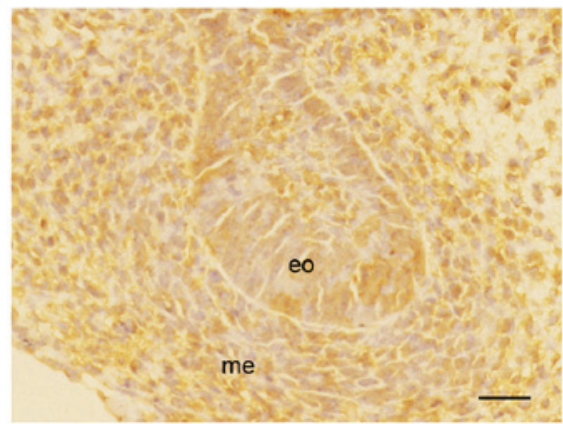

D

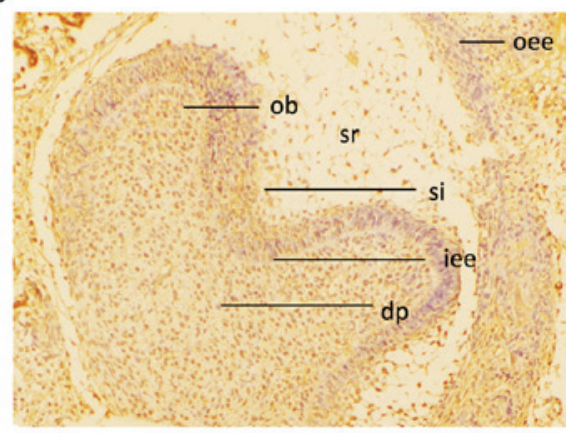

Figure 1. Immunostaining was used to detected the expression of SATB2 (brown coloration) in embryonic murine tooth germs at magnification, x200. (A) At the bud stage (E13.5), the dental lamina was thickened and invaginated into the underlying mesenchyme. SATB2 was expressed in the tooth bud cells and the underlying mesenchyme. (B) During the cap stage (E14.5), the enamel organ underwent further proliferation and differentiation. SATB2 was also detected in the enamel organ and mesenchyme. (C) At the early bell stage (E16.5), SATB2 staining was positive in the inner and outer enamel epithelium and the stratum intermedium; however, no expression was indicated in the stellate reticulum. Positive staining for SATB2 was also identified in the enamel knot. (D) At the late bell stage (E18.5), positive immunostaining was observed in odontoblasts and dental papilla cells. The differentiating ameloblasts, stratum intermedium and inner enamel epithelium were all stained SATB2-positive. Scale bar, $20 \mu \mathrm{m}$. eo, enamel organ; me, mesenchyme; iee, inner enamel epithelium; oee, outer enamel epithelium; sr, stellate reticulum; dp, dental papilla; ek, enamel knot; si, stratum intermedium; ob, odontoblasts; SATB2, special AT-rich sequence binding protein 2; E, embryonic day.
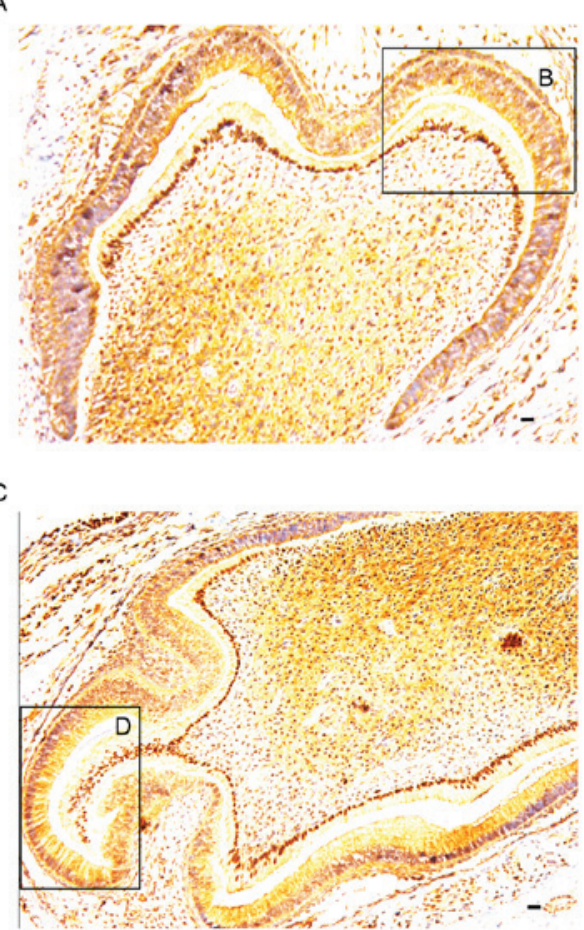
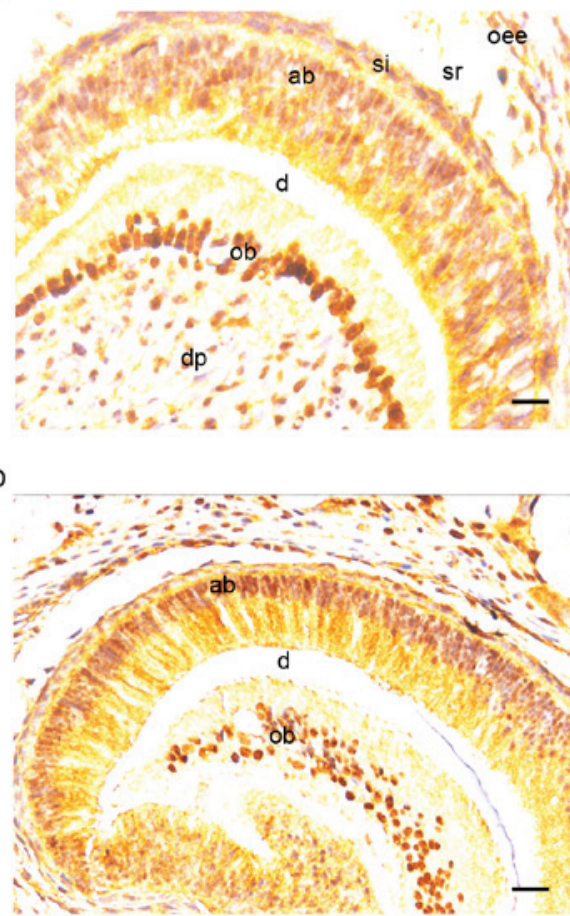

Figure 2. Immunostaining for SATB2 (brown coloration) in postnatal murine tooth germs at (A and B) P1 (magnification, $\mathrm{x} 40$ ) and (C and D) P5 (magnification, x200). At (A) P1 and (C) P5, positive staining was observed in the ameloblasts and intense SATB2-positive staining was present in the odontoblasts and dental pulp cells. (B and D) In the odontoblasts, marked SATB2 expression was detected in nuclei, whereas expression was predominantly in the cytosol and partially in nuclei in the ameloblasts. Scale bar, $20 \mu \mathrm{m}$. oee, outer enamel epithelium; sr, stellate reticulum; dp, dental pulp; si, stratum intermedium; ob, odontoblasts; ab ameloblasts, d dentin; SATB2, special AT-rich sequence binding protein 2; P, postnatal day. 
A

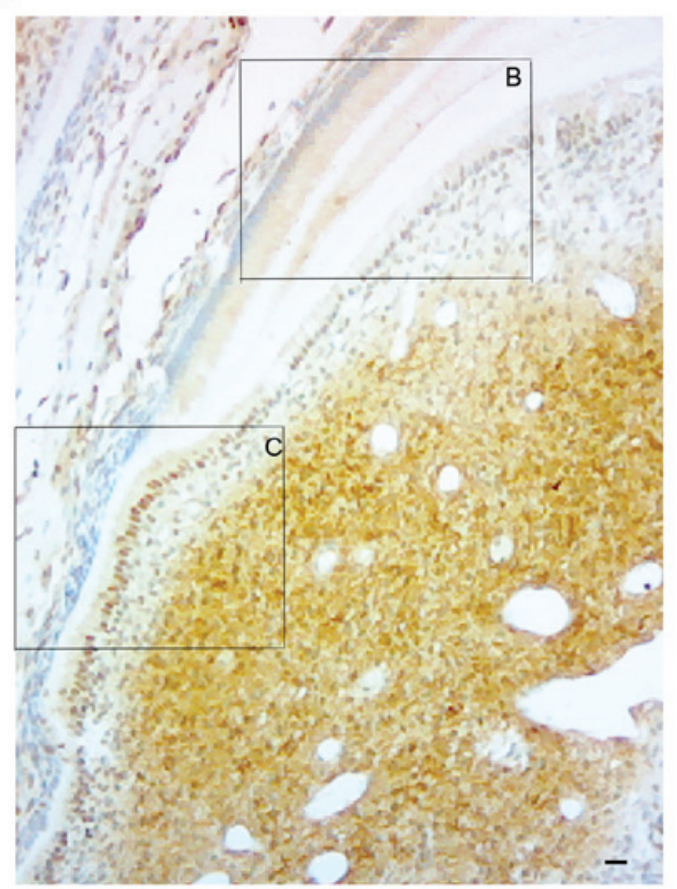

B
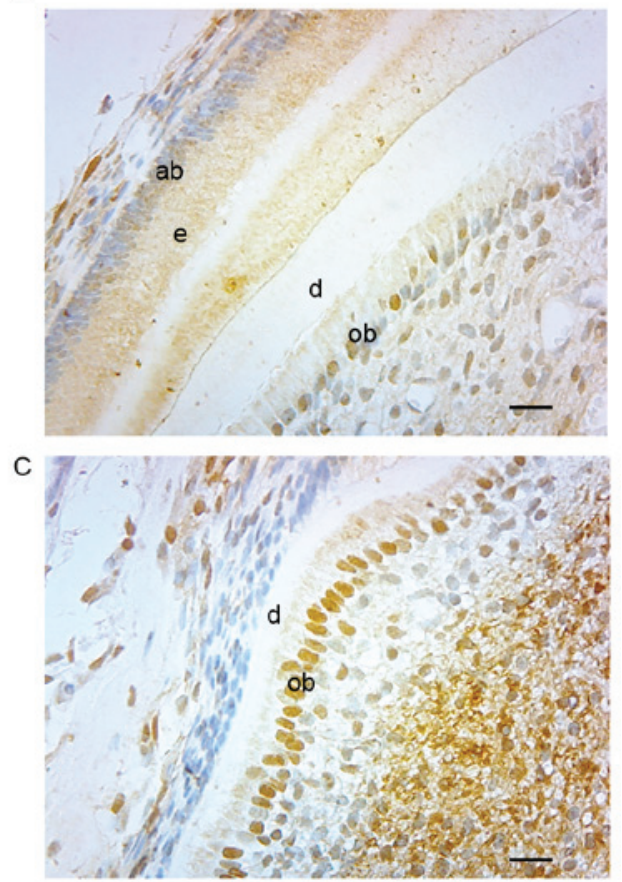

Figure 3. Immunostaining for SATB2 expression (brown coloration) in postnatal murine tooth germs at P7. (A) At P7, when the mineralized tissue was gradually deposited, SATB2 expression was still detected in the ameloblasts and dental pulp cells. Basic completion of crown morphogenesis was indicated and initial root development was observed. Magnification, x40. (B and C) On the pulpal side of root dentin, odontoblasts were lined in a row. SATB2 was still strongly expressed in the odontoblasts in the crown and in the root. Notably, SATB2-positive staining of odontoblasts in the root was more potent compared with that in the crown. Magnification, x200. Scale bar, $20 \mu \mathrm{m}$. ob, odontoblasts; ab, ameloblasts; e, enamel; d, dentin; SATB2, special AT-rich sequence binding protein 2; P, postnatal day.

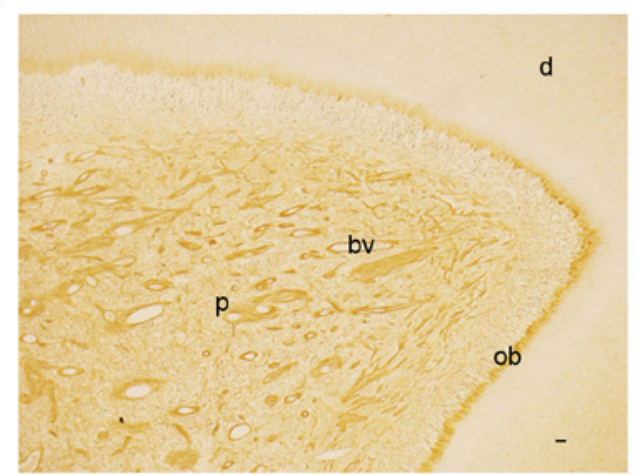

C

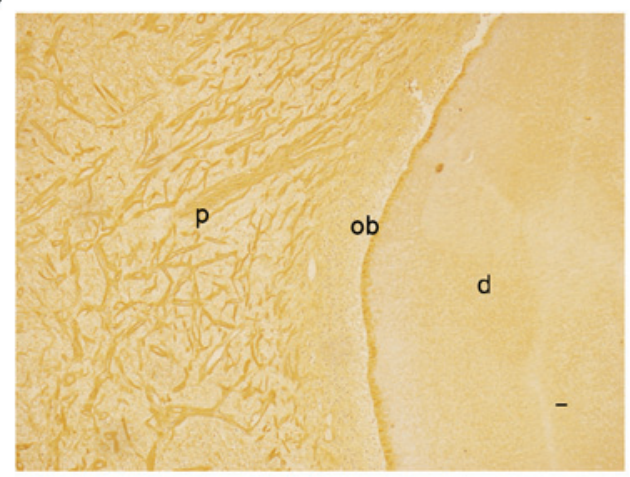

B

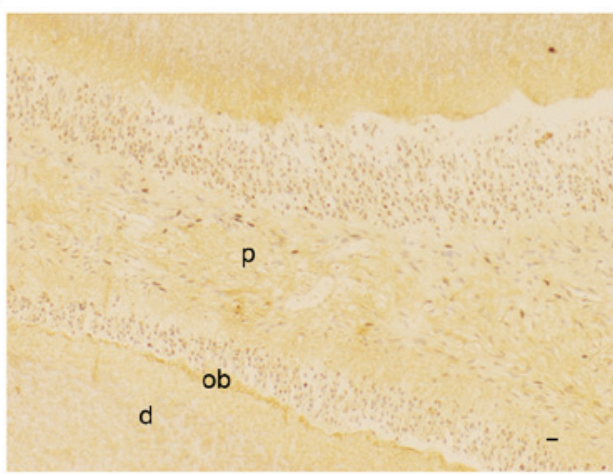

D

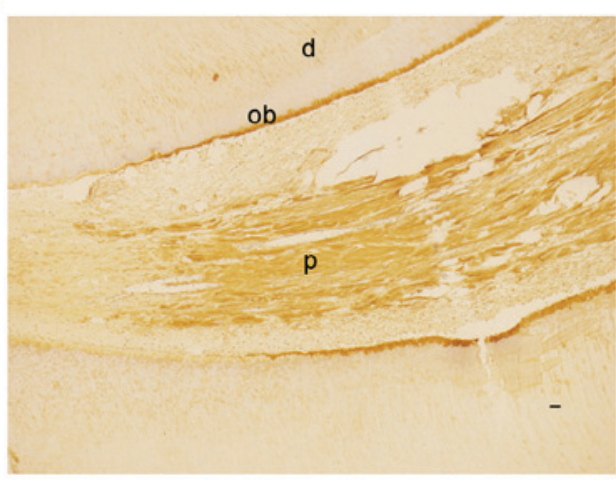

Figure 4. Immunostaining for SATB2 (brown coloration) in pulp-dentin complex of human healthy teeth and teeth with pulpitis at magnification x40. SATB2 was primarily localized in the odontoblasts and dental pulp of (A) crown and (B) root of healthy teeth and (C) crown and (D) root of teeth with pulpitis. However, immunohistochemical staining was more potent in the odontoblasts of the root in teeth with (D) pulpitis compared with (B) healthy teeth. In the odontoblasts of the teeth with pulpitis, the immunohistochemical staining was more potent in (D) the root compared with (C) the crown; however, the opposite was observed in (A and B) the healthy tooth. (A) In the healthy teeth, SATB2 expression was indicated in several blood vessels in the coronal pulp, (B) but not in any blood vessels in the pulp of root. Scale bar, $20 \mu \mathrm{m}$. ob, odontoblasts; d, dentin; bv, blood vessels; SATB2, special AT-rich sequence binding protein 2 . 
dental pulp in healthy teeth (Fig. 4A and B) and teeth with pulpitis (Fig. 4C and D). However, the immunohistochemical staining was more potent in the odontoblasts of the root in teeth with pulpitis (Fig. 4C and D) compared with healthy teeth (Fig. 4A and B). In addition, in the odontoblasts of the teeth with pulpitis, the immunohistochemical staining was more potent in the root compared with the crown; conversely, the opposite was indicated in the healthy teeth. Furthermore, in the healthy teeth, SATB2 was identified in some blood vessels in the coronal pulp (Fig. 4A), but not in any blood vessels in the pulp of the root (Fig. 4B).

\section{Discussion}

SATB2 is an important positive regulator in osteoblast differentiation, bone formation, and bone regeneration $(17,18)$. It has previously been demonstrated that SATB2 was able to repair and regenerate calvarial defects (19). In addition, SATB2 has also been reported to be expressed in the cortex of the brain, breast tissues, spinal cord, kidneys, umbilical cord, leukocytes and the central nervous system $(9,17)$, which suggests a distinct role of SATB2 in different tissues. To the best of our knowledge, the present study indicated for the first time the expression patterns of SATB2 during murine molar development from E13.5-P7 and in the pulp-dentin complex of human healthy teeth and teeth with pulpitis. The spatial and temporal expression of SATB2 indicated that SATB2 may have a role in tooth development and dentin formation.

At the bud and cap stages, SATB2 expression was detected in the whole enamel organ and underlying mesenchyme, which was consistent with a previous report that indicated SATB2 was expressed in the mesenchyme underlying the medial edge epithelia (20). The findings suggest that SATB2 may have a role in ensuring correct epithelial-mesenchymal interactions during the initiation stages of tooth development.

At the bell stage, intense SATB2-positive immunostaining was observed in odontoblasts and dental papilla cells, which suggested that SATB2 may participate in odontoblast differentiation and dentin formation.

During the postnatal day prior to tooth eruption, the expression of SATB2 in ameloblasts, odontoblasts and dental pulp cells indicated that SATB2 may have a role in the differentiation of ameloblasts and odontoblasts. Furthermore, when crown morphogenesis was completed and root development was initiated, the different expression levels of SATB2 in the odontoblasts between the root and the crown indicated that SATB2 was highly expressed in the region where odontoblast differentiation and dentin matrix mineralization occurred. Previous results have demonstrated that odontoblasts line the peripheral side of the dental pulp, produce the extracellular dentin matrix and are subsequently involved in the dentin mineralization process $(21,22)$. These findings suggested that SATB2 may not be essential once the mineralization process is completed, and also indicated that SATB2 may be involved in odontoblast differentiation and dentin formation.

In the present study, SATB2 immunoreactivity was detected in the odontoblasts and predentin, but not in the mineralized mature dentin of teeth. Previous results have indicated that SATB2 is associated with osteoblast differentiation and bone formation $(9,13,16)$. Similarly, the expression pattern of SATB2 in the human pulp-dentin complex and the absence of SATB2 in mature dentin in the present study indicated that SATB2 may participate in the odontoblast differentiation and dentin matrix mineralization during dentin formation.

During tooth development, odontoblasts are responsible for primary dentinogenesis (23). When suffering from unfavorable external stimuli (such as abrasion, etching and caries), odontoblasts and newly formed odontoblast-like cells derived from dental pulp stem cells are responsible for restorative dentinogenesis (24). Similar to that in murine molar development, SATB2 expression was highly expressed in the region where odontoblast differentiation and dentin matrix mineralization occurred, including in the root of teeth with pulpitis. This further suggested that SATB2 has a role in odontoblast differentiation and dentin matrix mineralization in healthy teeth and teeth with pulpitis. Furthermore, the present study indicated that SATB2 was expressed in the blood vessels, which suggests that SATB2 may be associated with the regulation of dental pulp physiological functions.

Previous studies have demonstrated that SATB2 regulates the expression and function of two vital genes associated with osteoblast differentiation and bone formation, Runx 2 and osterix (Osx), which have essential roles in bone and tooth development (25-30). Furthermore, a number of previous studies have indicated that SATB2 triggers Runx2 function, and interaction of SATB2 with Runx2 may be involved in the early stages of osteoblast differentiation $(9,17,31)$. In addition, a previous study has also suggested that SATB2 is a downstream target of Osx (32). Zhao et al (17) indicated that SATB2 upregulated Osx expression in a Runx2-independent manner and also synergistically enhanced the effect of Runx 2 on the Osx promoter. These findings imply that the expression and function of SATB2 in odontoblast differentiation and dentin formation may also be regulated by Runx 2 and Osx, which suggests a potential molecular mechanism of SATB2 in tooth development.

In conclusion, to the best of our knowledge, the present study is the first to indicate that SATB2 exhibited a spatiotemporal expression pattern in murine molar development and that SATB2 is expressed in odontoblasts, predentin, dental pulp cells and blood vessels in human teeth. These findings suggest that SATB2 may have important roles in odontoblast differentiation and dentin matrix mineralization during tooth development. However, the role of SATB2 and its precise mechanism during human tooth development requires further investigation.

\section{Acknowledgements}

The present study was supported by the Nature Science Foundation of China (grant nos. 81271132 and 81570963) and the Nature Science Foundation of Heilongjiang Province (grant no. H201440).

\section{References}

1. Thesleff I: Epithelial-mesenchymal signaling regulating tooth morphogenesis. J Cell Sci 116: 1647-1648, 2003. 
2. Viriot L, Peterkova R, Vonesch JL, Peterka M, Ruch JV and Lesot H: Mouse molar morphogenesis revisited by three-dimensional reconstruction. III. Spatial distribution of mitoses and apoptosis up to bell-staged first lower molar teeth. Int J Dev Biol 41: 679-690, 1997.

3. Chen Z, Couble ML, Mouterfi N, Magloire H, Chen Z and Bleicher F: Spatial and temporal expression of KLF4 and KLF5 during murine tooth development. Arch Oral Biol 54: 403-411, 2009.

4. Du J, Wang Q, Wang L, Wang X and Yang P: The expression pattern of FHL2 during mouse molar development. J Mol Histol 43: 289-295, 2012.

5. Fang P, Wang X, Zhang L, Yuan G, Chen Z and Zhang Q: Immunohistochemical localization of LIM mineralization protein 1 during mouse molar development. J Mol Histol 41: 199-203, 2010

6. Feng J, McDaniel JS, Chuang HH, Huang O, Rakian A, Xu X, Steffensen B, Donly KJ, Macdougall M and Chen S: Binding of amelogenin to MMP-9 and their co-expression in developing mouse teeth. J Mol Histol 43: 473-485, 2012.

7. Sloan AJ, Rutherford RB and Smith AJ: Stimulation of the rat dentine-pulp complex by bone morphogenetic protein-7 in vitro. Arch Oral Biol 45: 173-177, 2000.

8. Melin M, Joffre-Romeas A, Farges JC, Couble ML, Magloire H and Bleicher F: Effects of TGFbetal on dental pulp cells in cultured human tooth slices. J Dent Res 79: 1689-1696, 2000.

9. Dobreva G, Chahrour M, Dautzenberg M, Chirivella L, Kanzler B, Fariñas I, Karsenty G and Grosschedl R: SATB2 is a multifunctional determinant of craniofacial patterning and osteoblast differentiation. Cell 125: 971-986, 2006.

10. Zhang J, Tu Q, Grosschedl R, Kim MS, Griffin T, Drissi H, Yang P and Chen J: Roles of SATB2 in osteogenic differentiation and bone regeneration. Tissue Eng Part A 17: 1767-1776, 2011

11. Kim IS, Jeong SJ, Kim SH, Jung JH, Park YG and Kim SH: Special AT-rich sequence-binding protein 2 and its related genes play key roles in the differentiation of MC3T3-E1 osteoblast like cells. Biochem Biophys Res Commun 417: 697-703, 2012.

12. Britanova O, Akopov S, Lukyanov S, Gruss P and Tarabykin V: Novel transcription factor SATB2 interacts with matrix attachment region DNA elements in a tissue-specific manner and demonstrates cell-type-dependent expression in the developing mouse CNS. Eur J Neurosci 21: 658-668, 2005.

13. Ordonez NG: SATB2 is a novel marker of osteoblastic differentiation and colorectal adenocarcinoma. Adv Anat Pathol 21: 63-67, 2014.

14. Hu N, Feng C, Jiang Y, Miao Q and Liu H: Regulative effect of Mir-205 on osteogenic differentiation of bone mesenchymal stem cells (BMSCs): Possible role of SATB2/Runx 2 and ERK/MAPK pathway. Int J Mol Sci 16: 10491-10506, 2015.

15. Ellies DL and Krumlauf R: Bone formation: The nuclear matrix reloaded. Cell 125: 840-842, 2006.

16. Bleicher F, Couble ML, Farges JC, Couble P and Magloire H: Sequential expression of matrix protein genes in developingrat teeth. Matrix Biol 18: 133-143, 1999.

17. Zhao X, Qu Z, Tickner J, Xu J, Dai K and Zhang X: The role of SATB2 in skeletogenesis and human disease. Cytokine Growth Factor Rev 25: 35-44, 2014.
18. Gong Y, Qian Y, Yang F, Wang H and Yu Y: Lentiviral-mediated expression of SATB2 promotes osteogenic differentiation of bone marrow stromal cells in vitro and in vivo. Eur J Oral Sci 122: 190-197, 2014.

19. Zhang P, Men J, Fu Y, Shan T, Ye J, Wu Y, Tao Z, Liu L and Jiang H: Contribution of SATB2 to the stronger osteogenic potential of bone marrow stromal cells from craniofacial bones. Cell Tissue Res 350: 425-437, 2012.

20. FitzPatrick DR, Carr IM, McLaren L, Leek JP, Wightman P, Williamson K, Gautier P, McGill N, Hayward C, Firth H, et al: Identification of SATB2 as the cleft palate gene on 2q32-q33. Hum Mol Genet 12: 2491-2501, 2003.

21. Mjör IA, Sveen OB and Heyeraas KJ: Pulp-dentin biology in restorative dentistry. Part 1: Normal structure and physiology. Quintessence Int 32: 427-446, 2001

22. Smith AJ: Vitality of the dentin-pulp complex in health and disease: Growth factors as key mediators. J Dent Educ 67: 678-689, 2003.

23. Simon S, Smith AJ, Lumley PJ, Berdal A, Smith G, Finney S and Cooper PR: Molecular characterization of young and mature odontoblasts. Bone 45: 693-703, 2009.

24. Smith AJ, Murray PE, Sloan AJ, Matthews JB and Zhao S: Trans-dentinal stimulation of tertiary dentinogenesis. Adv Dent Res 15: 51-54, 2001.

25. Aberg T, Wang XP, Kim JH, Yamashiro T, Bei M, Rice R, Ryoo HM and Thesleff I: Runx2 mediates FGF signaling from epithelium to mesenchyme during tooth morphogenesis. Dev Biol 270: 76-93, 2004

26. Chen S, Rani S, Wu Y, Unterbrink A, Gu TT, Gluhak-Heinrich J, Chuang HH and Macdougall M: Differential regulation of dentin sialophosphoprotein expression by Runx 2 during odontoblast cytodifferentiation. J Biol Chem 280: 29717-29727, 2005.

27. Komori T: Regulation of bone development and extracellular matrix protein genes by UNX2. Cell Tissue Res 339: 189-195, 2010.

28. Li S, Kong H, Yao N, Yu Q, Wang P, Lin Y, Wang J, Kuang R, Zhao X, Xu J, et al: The role of runt-related transcription factor (Runx2) in the late stage of odontoblast differentiation and dentin formation. Biochem Biophys Res Commun 410: 698-704, 2011.

29. Chen S, Gluhak-Heinrich J, Wang YH, Wu YM, Chuang HH, Chen L, Yuan GH, Dong J, Gay I and MacDougall M: Runx2, osx, and dspp in tooth development. J Dent Res 88: 904-909, 2009.

30. Yang G, Li X, Yuan G, Liu P and Fan M: The effects of osterix on the proliferation and odontoblastic differentiation of human dental papilla cells. J Endod 40: 1771-1777, 2014.

31. Wei J, Shi Y, Zheng L, Zhou B, Inose H, Wang J, Guo XE, Grosschedl R and Karsenty G: miR-34s inhibit osteoblast proliferation and differentiation in the mouse by targeting SATB2. J Cell Biol 197: 509-521, 2012.

32. Tang W, Li Y, Osimiri L and Zhang C: Osteoblast-specific transcription factor Osterix (Osx) is an upstream regulator of Satb2 during bone formation. J Biol Chem 286: 32995-33002, 2011. 